\title{
Trade-off Relation among Genuine Three-qubit Nonlocalities in Four-qubit Systems
}

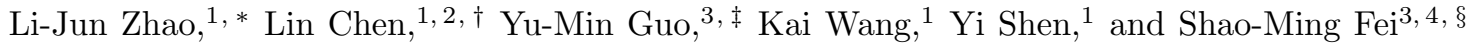 \\ ${ }^{1}$ School of Mathematics and Systems Science, Beihang University, Beijing 100191, China \\ ${ }^{2}$ International Research Institute for Multidisciplinary Science, Beihang University, Beijing 100191, China \\ ${ }^{3}$ School of Mathematical Sciences, Capital Normal University, Beijing 100048, China \\ ${ }^{4}$ Max-Planck-Institute for Mathematics in the Sciences, Leipzig 04103, Germany
}

\begin{abstract}
We study the trade-off relations satisfied by the genuine tripartite nonlocality in multipartite quantum systems. From the reduced three-qubit density matrices of the four-qubit generalized GHZ states and W states, we find that there exists a trade-off relation among the mean values of the Svetlichny operators associated with these reduced states. Namely, the genuine three-qubit nonlocalities are not independent. For four-qubit generalized GHZ states and W states, the summation of all their three-qubit maximal (squared) mean values of the Svetlichny operator is upper bounded. And this bound is superior to the one derived from the upper bounds of individual three-qubit mean values of the Svetlichny operator. Detailed examples are presented to illustrate the trade-off relation among the three-qubit nonlocalities.
\end{abstract}

PACS numbers: 03.65.Ud, 03.67.Mn

\section{INTRODUCTION}

Nonlocality is a fundamental feature of quantum mechanics 1, 2. It is also a key resource in information processing [3 6], and is related to various topics in quantum information theory such as the understanding of classical and quantum boundary [7, 8], the entangling power of nonlocal unitary operations 9 11, and the efficient decomposition for realization in quantum circuits [12, unextendible product basis [13, and positivepartial-transpose entangled states [14].

Bell inequalities and nonlocality have been widely studied and are shown to be related to the monogamy trade-off obeyed by bipartite Bell correlations. It is believed that for general translation invariant systems, twoqubit states should not violate the Bell inequality [15]. A nontrivial model is constructed to confirm that the Bell inequality can be violated in perfect translation-invariant systems with an even number of sites [16. Monogamy relations between the violations of Bell's inequalities have been derived in [17. Meanwhile, using the Bloch vectors, a trade-off relation has been derived, together with a complete classification of four-qudit quantum states [18].

In the multipartite case, nonlocality displays a much richer and more complex structure compared with the case of bipartite systems. This makes the study and the characterization of multipartite nonlocal correlations an interesting, but challenging problem. It comes thus to no surprise that our understanding of nonlocality in the multipartite setting is much less advanced than in the bipartite case [19, 20].

In 21] a complete characterization of entanglement of

lijunzhao@buaa.edu.cn

† linchen@buaa.edu.cn (corresponding author)

guo ym@cnu.edu.cn (corresponding author)

$\S$ feishm@cnu.edu.cn (corresponding author) an entire class of mixed three-qubit states with the same symmetry as the Greenberger-Horne-Zeilinger state, known as GHZ-symmetric states, has been achieved. By analytical expressions of maximum violation value of most efficient Bell inequalities one obtains the conditions of standard nonlocality and genuine nonlocality of this class of states. The relation between entanglement and nonlocality has been also discussed for this class of states. Interestingly, genuine entanglement of GHZ-symmetric states is necessary to reveal the standard nonlocality 22. Nonlocal correlations are proposed in three-qubit generalized GHZ states and four-qubit generalized GHZ states 23. Meanwhile, all multipartite pure states that are equivalent to the $\mathrm{N}$-qubit $\mathrm{W}$ states under stochastic local operation and classical communication (SLOCC) can be uniquely determined (among arbitrary states) from their bipartite marginals [24].

Two overlapping bipartite binary Bell inequalities cannot be simultaneously violated, which would contradict the usual no-signaling principle. It is known as the monogamy of Bell inequality violations. Generally Bell monogamy relations refer to trade-offs between simultaneous violations of multiple inequalities. The genuine multipartite nonlocality, as evidenced by a generalized Svetlichny inequality, does exhibit monogamy property 25. There is a complementarity relation between dichotomic observables leading to the monogamy of Bell inequality violations [26].

To study the nonlocality of bipartite quantum states, one considers the Clauser-Horne-Shimony-Holt (CHSH) inequality [27]. For any two-qubit density matrix $\rho$, if there exist local hidden variable models to describe the system, the CHSH inequality says that

$$
\left|\operatorname{Tr}\left(\rho B_{C H S H}\right)\right| \leq 2,
$$

where $B_{C H S H}$ is the CHSH operator

$$
B_{C H S H}=\vec{a} \cdot \vec{\sigma} \otimes\left(\vec{b}+\vec{b}^{\prime}\right) \cdot \vec{\sigma}+\vec{a}^{\prime} \cdot \vec{\sigma} \otimes\left(\vec{b}-\vec{b}^{\prime}\right) \cdot \vec{\sigma},
$$


with $\vec{a}, \vec{a}^{\prime}, \vec{b}$ and $\vec{b}^{\prime}$ the real three-dimensional unit vectors, and $\vec{\sigma}=\left(\sigma_{1}, \sigma_{2}, \sigma_{3}\right)$ the Pauli matrices. Denote $T$ the matrix with entries given by $t_{i j}=\operatorname{Tr}\left[\rho\left(\sigma_{i} \otimes \sigma_{j}\right]\right.$. It has been shown that the maximal violation of the CHSH inequality (1) is given by 28,29 ,

$$
\langle C H S H\rangle_{\rho}=\max \left|\operatorname{Tr}\left(\rho B_{C H S H}\right)\right|=2 \sqrt{M(\rho)},
$$

where $M(\rho)=\max _{j<k}\left\{\mu_{j}+\mu_{k}\right\}, j, k \in\{1,2,3\}, \mu_{j}, \mu_{k}$ are the two largest eigenvalues of the real symmetric matrix $T^{t} T$, and $t$ denotes the matrix transposition.

The distribution of nonlocality in multipartite systems based on the violation of Bell inequality has been investigated in 30, 31. For any 3 -qubit state $\rho_{A B C} \in$ $\mathcal{H}^{A} \otimes \mathcal{H}^{B} \otimes \mathcal{H}^{C}$, the maximal violation of CHSH inequality of pairwise bipartite states satisfies the following tradeoff relation:

$$
\langle C H S H\rangle_{\rho_{A B}}^{2}+\langle C H S H\rangle_{\rho_{A C}}^{2}+\langle C H S H\rangle_{\rho_{B C}}^{2} \leq 12 .
$$

It implies that for a three qubit system, it is impossible that all pairs of qubit states violate the CHSH inequality simultaneously.

For genuine tripartite nonlocality, consider three separated observers Alice, Bob and Charlie, with their measurement settings $x, y, z$ and outputs $a, b, c$, respectively. The correlations are said to be local if the joint probability distribution $p(a b c \mid x y z)$ can be written as

$$
p(a b c \mid x y z)=\int d \lambda q(\lambda) p_{\lambda}(a \mid x) p_{\lambda}(b \mid y) p_{\lambda}(c \mid z),
$$

where $\lambda$ is the local random variable and $\int d \lambda q(\lambda)=1$. A state is called genuine tripartite non-local if $p(a b c \mid x y z)$ can not be written as

$$
\begin{aligned}
p(a b c \mid x y z)= & \int d \lambda q(\lambda) p_{\lambda}(a b \mid x y) p_{\lambda}(c \mid z) \\
& +\int d \mu q(\mu) p_{\mu}(b c \mid y z) p_{\mu}(a \mid x) \\
& +\int d \nu q(\nu) p_{\nu}(a c \mid x z) p_{\nu}(b \mid y),
\end{aligned}
$$

where $\int d \lambda q(\lambda)+\int d \mu q(\mu)+\int d \nu q(\nu)=1$. A state satisfying (4) is said to admit bi-LHV (local hidden variable) model. Svetlichny introduced an inequality to verify the genuine tripartite nonlocality. There are also two alternative definitions of n-way nonlocality, and a series of Bell-type inequalities for the detection of three-way nonlocality [32]. Nevertheless, such $n$ - way nonlocalities are strictly weaker than the Svetlichny's. The dynamics of the nonlocality measured by the violation of Svetlichny's Bell-type inequality has been investigated in the nonMarkovian model [33.

To quantify the nonlocality of three-qubit states, in [34, a technique is developed to find the maximal violation of the Svetlichny inequality, and a tight upper bound is obtained. In this paper, we explicitly quantify the genuine tripartite nonlocality of the reduced states of four-qubit pure states. We first introduce the Svetlichny inequality whose violation is a signature of the genuine tripartite nonlocality. According to the maximal value of the Svetlichny operator we show that there exists a tradeoff relation among the mean values of the Svetlichny operators associated with the three-qubit reduced states of GHZ and W states. We present detailed examples to illustrate the trade-off relation among such genuine threequbit nonlocalities. The rest of this paper is organized as follows. In Sec. III we introduce the Svetlichny inequality. In Sec. III and IV we investigate the trade-off for four-qubit symmetric pure states in the space spanned by Dicke states. Finally we conclude in Sec. V]

\section{SVETLICHNY INEQUALITY}

We consider the nonlocality test scenario for threequbit systems associated with Alice, Bob and Chalie. Let the two measurement observables for Alice be $A=\vec{a} \cdot \vec{\sigma}$ and $A^{\prime}=\vec{a}^{\prime} \cdot \vec{\sigma}$, where $\vec{a}$ and $\vec{a}^{\prime}$ are unit vectors in $\mathbb{R}^{3}$, and $\vec{\sigma}=\left(\sigma_{1}, \sigma_{2}, \sigma_{3}\right)$ is the vector of Pauli matrices. Each observable is an Hermitian operator with eigenvalues \pm 1 . Similarly, we have $B=\vec{b} \cdot \vec{\sigma}$ and $B^{\prime}=\overrightarrow{b^{\prime}} \cdot \vec{\sigma}$ for Bob, and $C=\vec{c} \cdot \vec{\sigma}$ and $C^{\prime}=\vec{c}^{\prime} \cdot \vec{\sigma}$ for Charlie. The Svetlichny operator corresponding to measurements $A, A^{\prime}, B, B^{\prime}, C$ and $C^{\prime}$ is defined by

$$
\begin{aligned}
S:= & A\left(\left(B+B^{\prime}\right) C+\left(B-B^{\prime}\right) C^{\prime}\right) \\
& +A^{\prime}\left(\left(B-B^{\prime}\right) C-\left(B+B^{\prime}\right) C^{\prime}\right) \\
= & A\left(D C+D^{\prime} C^{\prime}\right)+A^{\prime}\left(D^{\prime} C-D C^{\prime}\right),
\end{aligned}
$$

where $D=B+B^{\prime}$ and $D^{\prime}=B-B^{\prime}$.

If a 3-qubit state $\rho$ admits a bi-LHV model, then it satisfies the Svetlichny inequality [35],

$$
\langle S(\rho)\rangle=\operatorname{Tr}(S \rho) \leq 4,
$$

for all possible Svetlichny operators $S$. Conversely, a 3qubit state which violates this inequality for some $S$ is genuine three-qubit nonlocal. To quantify the nonlocality of a 3-qubit system, we need to compute the maximum of the so-called Svetlichny value,

$$
S_{\text {max }}(\rho)=\max \operatorname{Tr}(S \rho),
$$

where the maximization is taken over all possible Svetlichny operators. Thus, $S_{\max }(\rho)>4$ is a sufficient condition for $\rho$ to be genuine three-qubit nonlocal. Moreover, the maximal Svetlichny value is $4 \sqrt{2}$ when the Svetlichny inequality is maximally violated by, say, the GHZ state $(|000\rangle+|111\rangle) / \sqrt{2}[35$, 36]. It has been shown in 34 that for any three-qubit state $\rho$, the maximal value $S_{\max }$ related to the Svetlichny operator $S$ satisfies

$$
S_{\max }(\rho) \leq 4 \lambda_{1},
$$

where $\lambda_{1}$ is the maximum singular value of the matrix $M=\left(m_{j, i k}\right)$, with $m_{i j k}=\operatorname{Tr}\left(\rho\left(\sigma_{i} \otimes \sigma_{j} \otimes \sigma_{k}\right)\right), i, j, k=$ $1,2,3$. 


\section{TRADE-OFF RELATIONS WITH RESPECT TO FOUR-QUBIT SYMMETRIC STATES}

Let $\vec{x}=\left(\sin \theta_{x} \cos \phi_{x}, \sin \theta_{x} \sin \phi_{x}, \cos \theta_{x}\right)$ for $x=$ $a, a^{\prime}, b, b^{\prime}, c, c^{\prime}$. Set $\vec{b}+\overrightarrow{b^{\prime}}=2 \vec{d} \cos \omega$ and $\vec{b}-\vec{b}^{\prime}=2 \overrightarrow{d^{\prime}} \sin \omega$. If $\omega \neq \pi n / 2$ for $n \in \mathbb{Z}, \vec{b}+\vec{b}^{\prime}$ and $\vec{b}-\vec{b}^{\prime}$ are mutually orthogonal. If $\omega=\pi n / 2$ for $n \in \mathbb{Z}$, for example, $\omega=\pi / 2$, then $\overrightarrow{d^{\prime}}=\vec{b}$. We can still construct a $\vec{d}$ which is orthogonal to $\overrightarrow{d^{\prime}}$ in this case. These two vectors $\vec{d}$ and $\overrightarrow{d^{\prime}}$ satisfy

$$
\vec{d} \cdot \overrightarrow{d^{\prime}}=\cos \theta_{d} \cos \theta_{d^{\prime}}+\sin \theta_{d} \sin \theta_{d^{\prime}} \cos \left(\phi_{d}-\phi_{d^{\prime}}\right)=0,
$$

that is, the maximum of $\cos ^{2} \theta_{d}+\cos ^{2} \theta_{d^{\prime}}$ is 1 , while the maximum of $\sin ^{2} \theta_{d}+\sin ^{2} \theta_{d^{\prime}}$ is 2 . Then setting $D=\vec{d} \cdot \vec{\sigma}$ and $D^{\prime}=\vec{d} \cdot \vec{\sigma}$, we have

$$
\begin{gathered}
\langle S(\rho)\rangle=2 \mid \cos \omega\langle A D C\rangle_{\rho}+\sin \omega\left\langle A D^{\prime} C^{\prime}\right\rangle_{\rho} \\
+\sin \omega\left\langle A^{\prime} D^{\prime} C\right\rangle_{\rho}-\cos \omega\left\langle A^{\prime} D C^{\prime}\right\rangle_{\rho} \mid \\
\leq 2 \mid\left(\langle A D C\rangle_{\rho}^{2}+\left\langle A D^{\prime} C^{\prime}\right\rangle_{\rho}^{2}\right)^{1 / 2} \\
+\left(\left\langle A^{\prime} D^{\prime} C\right\rangle_{\rho}^{2}+\left\langle A^{\prime} D C^{\prime}\right\rangle_{\rho}^{2}\right)^{1 / 2} \mid,
\end{gathered}
$$

where the following inequality has been taken into account,

$$
x \cos \omega+y \sin \omega \leq\left(x^{2}+y^{2}\right)^{1 / 2},
$$

with the equality holds when $\tan \omega=\frac{y}{x}$, $x \cos \omega \geq 0, x \neq 0$; or $\sin \omega= \pm 1, y \sin \omega \geq 0, x=0$. (10) will be used in the following derivations.

Let us consider the four-qubit generalized Greenberger-Horne-Zeilinger (GGHZ) state $\left|\psi_{a b c d}\right\rangle$ and the generalized maximal slice (MS) state $\left|\phi_{a b c d}\right\rangle$ :

$$
\begin{aligned}
& \left|\psi_{a b c d}\right\rangle=\cos \theta|0000\rangle+\sin \theta|1111\rangle, \\
& \left|\phi_{a b c d}\right\rangle=\frac{1}{\sqrt{2}}|0000\rangle+\frac{1}{\sqrt{2}}|111\rangle(\cos \theta|0\rangle+\sin \theta|1\rangle) .
\end{aligned}
$$

Denote $\Psi_{a b c d}=\left|\psi_{a b c d}\right\rangle\left\langle\psi_{a b c d}\right|$ and $\Phi_{a b c d}=\left|\phi_{a b c d}\right\rangle\left\langle\phi_{a b c d}\right|$ the corresponding density matrices.

Theorem 1 For four-qubit GGHZ state $\Psi_{a b c d}=$ $\left|\psi_{a b c d}\right\rangle\left\langle\psi_{a b c d}\right|$, the violation of the Svetlichny inequality on any three-qubit states satisfies the following relation:

$\left\langle S\left(\Psi_{a b c}\right)\right\rangle+\left\langle S\left(\Psi_{a b d}\right)\right\rangle+\left\langle S\left(\Psi_{a c d}\right)\right\rangle+\left\langle S\left(\Psi_{b c d}\right)\right\rangle \leq 16|\cos 2 \theta|$,

where $\Psi_{a b c}=\Psi_{a b d}=\Psi_{a c d}=\Psi_{b c d}=\cos ^{2} \theta|000\rangle\langle 000|+$ $\sin ^{2} \theta|111\rangle\langle 111|$ are the corresponding reduced threequbit states. The equality holds in (13) when

$\left|\cos \theta_{a} \cos \theta_{c}-\cos \theta_{a^{\prime}} \cos \theta_{c^{\prime}}\right|=2, \omega=\theta_{d}=0, \theta_{d^{\prime}}=\pi / 2$.

Proof. By straightforward computation, we have

$$
\langle A D C\rangle_{\Psi_{a b c}}=\cos 2 \theta \cos \theta_{a} \cos \theta_{c} \cos \theta_{d},
$$

and similar expressions for $\left\langle A D^{\prime} C^{\prime}\right\rangle_{\Psi_{a b c}},\left\langle A^{\prime} D^{\prime} C\right\rangle_{\Psi_{a b c}}$ and $\left\langle A^{\prime} D C^{\prime}\right\rangle_{\Psi_{a b c}}$. From (10), we have

$$
\begin{array}{rl}
\left\langle S\left(\Psi_{a b c}\right)\right\rangle= & 2 \mid \cos \omega\langle A D C\rangle_{\Psi_{a b c}}+\sin \omega\left\langle A D^{\prime} C^{\prime}\right\rangle_{\Psi_{a b c}} \\
& +\sin \omega\left\langle A^{\prime} D^{\prime} C\right\rangle_{\Psi_{a b c}}-\cos \omega\left\langle A^{\prime} D C^{\prime}\right\rangle_{\Psi_{a b c}} \mid \\
\leq 2 & 2 \mid\left(\langle A D C\rangle_{\Psi_{a b c}}^{2}+\left\langle A D^{\prime} C^{\prime}\right\rangle_{\Psi_{a b c}}^{2}\right)^{1 / 2} \\
& +\left(\left\langle A^{\prime} D^{\prime} C\right\rangle_{\Psi_{a b c}}^{2}+\left\langle A^{\prime} D C^{\prime}\right\rangle_{\Psi_{a b c}}^{2}\right)^{1 / 2} \mid \\
= & 2 \mid \cos 2 \theta \cos \theta_{a}\left(\cos ^{2} \theta_{c} \cos ^{2} \theta_{d}\right. \\
& \left.+\cos ^{2} \theta_{c^{\prime}} \cos ^{2} \theta_{d^{\prime}}\right)^{\frac{1}{2}} \\
& +\cos 2 \theta \cos \theta_{a^{\prime}}\left(\cos ^{2} \theta_{c} \cos ^{2} \theta_{d^{\prime}}\right. \\
& \left.+\cos ^{2} \theta_{c^{\prime}} \cos ^{2} \theta_{d}\right)^{\frac{1}{2}} \mid
\end{array}
$$

Since the maximum of $\cos ^{2} \theta_{d}+\cos ^{2} \theta_{d^{\prime}}$ is 1 [37, the above formula can be further reduced to be,

$$
\left\langle S\left(\Psi_{a b c}\right)\right\rangle \leq 2|\cos 2 \theta|\left(\left|\cos \theta_{a}\right|+\left|\cos \theta_{a^{\prime}}\right|\right) \leq 4|\cos 2 \theta| .
$$

Since $\left\langle S\left(\Psi_{a b c}\right)\right\rangle=\left\langle S\left(\Psi_{a b d}\right)\right\rangle=\left\langle S\left(\Psi_{a c d}\right)\right\rangle=\left\langle S\left(\Psi_{b c d}\right)\right\rangle \leq$ $4|\cos 2 \theta|$ for the state $\Psi_{a b c d}=\left|\psi_{a b c d}\right\rangle\left\langle\psi_{a b c d}\right|$, one gets the inequality (13).

The equality holds in 13 when

$\left|\cos \theta_{a} \cos \theta_{c}-\cos \theta_{a^{\prime}} \cos \theta_{c^{\prime}}\right|=2, \omega=\theta_{d}=0, \theta_{d^{\prime}}=\pi / 2$,

namely, $S_{\max }\left(\Psi_{a b c}\right)=S_{\max }\left(\Psi_{a b d}\right)=S_{\max }\left(\Psi_{a c d}\right)=$ $S_{\max }\left(\Psi_{b c d}\right)=4|\cos 2 \theta| \leq 4$. It means that in this case all the reduced states of GGHZ state do not violate the Svetlichny inequality.

For the GGHZ state, the four reduced three-qubit states are the same. From (8), the maximal value of the Svetlichny operator is $4 \max \left\{\cos ^{4} \theta, \sin ^{4} \theta\right\}$ for any one of such reduced three-qubit states. It is remarkable that the upper bound in (13) is always less or equal to the upper bound $16 \max \left\{\cos ^{4} \theta, \sin ^{4} \theta\right\}$ derived from (8), see Figure 1 for $\theta \in\left[0, \frac{\pi}{4}\right]$.

Generalizing Theorem 1 to general $n$-qubit case, we have for $n \geq 4$

Corollary 1 For $n$-qubit GGHZ state $|\Psi\rangle=$ $\cos \theta|00 \cdots 0\rangle+\sin \theta|11 \cdots 1\rangle$, the violation of the Svetlichny inequality on any three-qubit states satisfies the following relation:

$$
\sum_{1 \leq I<J<K \leq n}\left\langle S\left(\Psi_{I J K}\right)\right\rangle \leq 4\left(\begin{array}{c}
n \\
3
\end{array}\right)|\cos 2 \theta|,
$$

where $\Psi_{I J K}=\operatorname{Tr}_{\overline{I J K}}|\Psi\rangle\left\langle\Psi\left|=\cos ^{2} \theta\right| 000\right\rangle\left\langle\left. 000\right|_{I J K}+\right.$ $\sin ^{2} \theta|111\rangle\left\langle\left. 111\right|_{I J K}\right.$ are the corresponding reduced threequbit states associated with qubits $I, J$ and $K$, and $\operatorname{Tr}_{\overline{I J K}}$ stands for the trace over the rest qubit systems.

Theorem 2 For four-qubit generallized MS states $\Phi_{a b c d}$, the violation of the Svetlichny inequality on the 


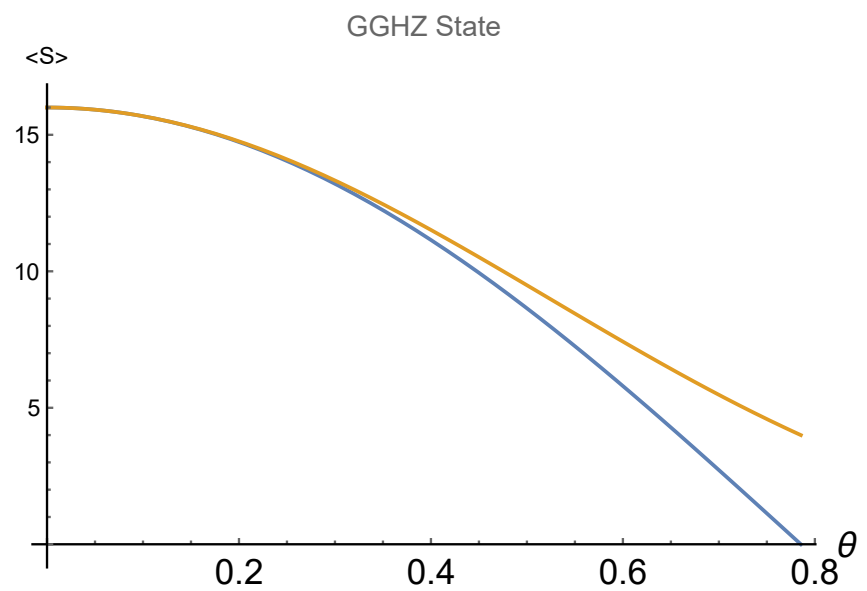

Figure 1: For $\theta \in\left[0, \frac{\pi}{4}\right]$, the upper bound of the sum of violations of the Svetlichny inequality for four reduced three-qubit states is $16|\cos 2 \theta|$. It is less or equal to $16 \max \left\{\cos ^{4} \theta, \sin ^{4} \theta\right\}=16 \cos ^{4} \theta$ derived from (8). The blue line is the bound from Theorem 1 . The yellow one comes from (8). When $\theta=0$, two bounds are equal.

reduced three-qubit density matrices satisfies the following relation:

$$
\begin{aligned}
& \left\langle S\left(\Phi_{a b c}\right)\right\rangle+\left\langle S\left(\Phi_{a b d}\right)\right\rangle+\left\langle S\left(\Phi_{a c d}\right)\right\rangle+\left\langle S\left(\Phi_{b c d}\right)\right\rangle \\
& \leq 4 \sqrt{2}|\cos \theta|+12\left|\cos ^{2} \theta+\frac{1}{2} \sin 2 \theta\right|,
\end{aligned}
$$

where

$$
\begin{aligned}
\Phi_{a b c}= & \frac{1}{2}|000\rangle\left\langle 000\left|+\frac{1}{2} \cos \theta\right| 000\right\rangle\langle 111| \\
& +\frac{1}{2} \cos \theta|111\rangle\left\langle 000\left|+\frac{1}{2}\right| 111\right\rangle\langle 111|, \\
\Phi_{a b d}= & \Phi_{a c d}=\Phi_{b c d} \\
= & \frac{1}{2}|000\rangle\left\langle 000\left|+\frac{1}{2} \cos ^{2} \theta\right| 110\right\rangle\langle 110| \\
& +\frac{1}{2} \cos \theta \sin \theta|110\rangle\left\langle 111\left|+\frac{1}{2} \cos \theta \sin \theta\right| 111\right\rangle\langle 110| \\
& +\frac{1}{2} \sin ^{2} \theta|111\rangle\langle 111| .
\end{aligned}
$$

See proof in Appendix A.

Inequality 18 gives a trade off relation of among the three-qubit genuine nonlocalities in MS states. In fact, by using (8) for any three-qubit states of a MS state, one has

$\left\langle S\left(\Phi_{a b c}\right)\right\rangle+\left\langle S\left(\Phi_{a b d}\right)\right\rangle+\left\langle S\left(\Phi_{a c d}\right)\right\rangle+\left\langle S\left(\Phi_{b c d}\right)\right\rangle \leq 20 \cos ^{2} \theta$.

Nevertheless, the upper bound of $(20)$ is larger than the one of 18$)$, see Figure 2 for $\theta \in(\pi / 2,3 \pi / 2)$.

Now consider the $n$-qubit generalized MS states,

$$
\left|\Psi_{12 \ldots n}\right\rangle=\frac{1}{\sqrt{2}}|00 \cdots 0\rangle+\frac{1}{\sqrt{2}}|11 \cdots 1\rangle|\psi\rangle,
$$

where $|\psi\rangle=\cos \theta|0\rangle+\sin \theta|1\rangle$. Let $\mathcal{I}$ denote a proper subset of $\{1,2, \ldots, n\}$. We define the states with $n \notin \mathcal{I}$

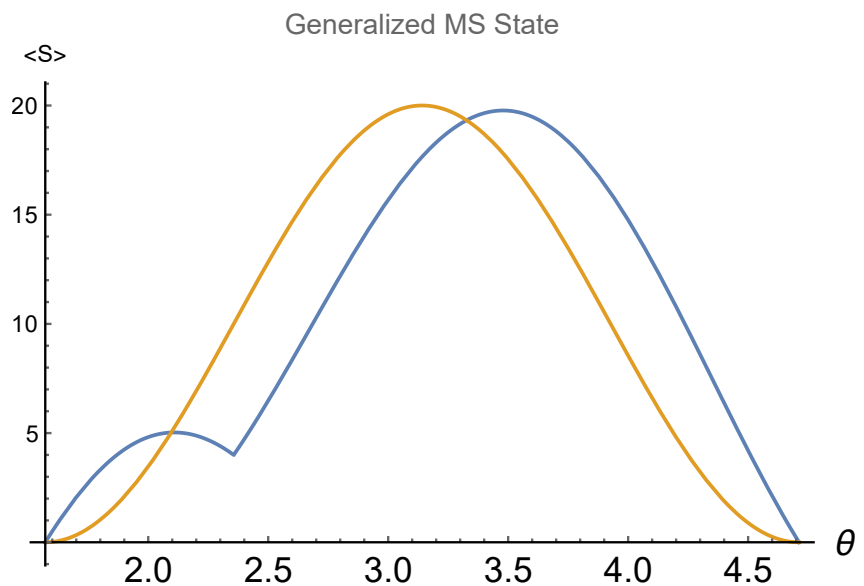

Figure 2: The blue line is for the upper bound of (18), the yellow line is for the upper bound of 20 for

$$
\theta \in(\pi / 2,3 \pi / 2) \text {. }
$$

the Class I $(\# \mathcal{I}=m<n)$, and $n \in \mathcal{I}$ the Class II. Then, there are $\left(\left(\begin{array}{c}n \\ m\end{array}\right)-\left(\begin{array}{c}n \\ m-1\end{array}\right)\right)$ states in class I, and $\left(\begin{array}{c}n \\ m-1\end{array}\right)$ states in class II,

$$
\rho_{\mathcal{I}}= \begin{cases}\frac{1}{2}|0 \cdots 00\rangle\left\langle 0 \cdots 00\left|+\frac{1}{2}\right| 1 \cdots 11\right\rangle\langle 1 \cdots 11| & n \notin \mathcal{I} \\ \frac{1}{2}|0 \cdots 00\rangle\left\langle 0 \cdots 00\left|+\frac{1}{2}\right| 1 \cdots 1 \psi\right\rangle\langle 1 \cdots 1 \psi| & n \in \mathcal{I}\end{cases}
$$

From Theorem 2 we have the following corollary,

Corollary 2 For $n$-qubit generallized MS states $\Phi_{a b c d}$, the violation of the Svetlichny inequality on the reduced three-qubit density matrices satisfies the following relation:

$$
\begin{aligned}
& \sum_{1 \leq I<J<K \leq n}\left\langle S\left(\Psi_{I J K}\right)\right\rangle \\
& \leq 4 \sqrt{2}\left(\begin{array}{c}
n-1 \\
2
\end{array}\right)|\cos \theta| \\
&+4\left(\left(\begin{array}{c}
n \\
3
\end{array}\right)-\left(\begin{array}{c}
n-1 \\
2
\end{array}\right)\right)\left|\cos ^{2} \theta+\frac{1}{2} \sin 2 \theta\right|,
\end{aligned}
$$

where $\Psi_{I J K}=\operatorname{Tr}_{\overline{I J K}}|\Psi\rangle\left\langle\Psi\left|=\frac{1}{2}\right| 000\right\rangle\left\langle\left. 000\right|_{I J K}+\right.$ $\frac{1}{2}|111\rangle\left\langle\left. 111\right|_{I J K}\right.$ for $\Psi_{I J K}$ belonging to Class $\mathrm{I}$, and $\Psi_{I J K}=\operatorname{Tr}_{\overline{I J K}}|\Psi\rangle\left\langle\Psi\left|=\frac{1}{2}\right| 000\right\rangle\langle 000|+$ $\frac{1}{2} \cos ^{2} \theta|110\rangle\left\langle 110\left|+\frac{1}{2} \cos \theta \sin \theta\right| 110\right\rangle\langle 111|+$ $\frac{1}{2} \cos \theta \sin \theta|111\rangle\left\langle 110\left|+\frac{1}{2} \sin ^{2} \theta\right| 111\right\rangle\langle 111|$ for $\Psi_{I J K}$ belonging to Class II.

\section{TRADE-OFF RELATIONS FOR THE W-CLASS STATES}

For a 4-qubit state:

$|\varphi\rangle_{a b c d}=\alpha|1000\rangle+\beta|0100\rangle+\gamma|0010\rangle+\delta|0001\rangle+\lambda|0000\rangle$,

with $\alpha, \beta, \gamma, \delta, \lambda$ are real numbers. It can generate fourqubit quantum states by unitary operators. We consider trade-off relation between the reduced states of $\left|\psi_{a b c d}\right\rangle$. 
Theorem 3 For any 4-qubit state $|\varphi\rangle_{a b c d}$, the violation of Svetlichny operators on tripartite states satisfies the following relation:

$$
\begin{aligned}
& \left\langle S\left(\rho_{a b c}\right)\right\rangle+\left\langle S\left(\rho_{a b d}\right)\right\rangle+\left\langle S\left(\rho_{a c d}\right)\right\rangle+\left\langle S\left(\rho_{b c d}\right)\right\rangle \\
& \leq 2\left(\left(2 x_{1}+8 y_{1}\right)^{\frac{1}{2}}+\left(2 x_{1}+8 y_{1}+8 \beta^{2} \gamma^{2}\right)^{\frac{1}{2}}\right) \\
& +2\left(\left(2 x_{2}+8 y_{2}\right)^{\frac{1}{2}}+\left(2 x_{2}+8 y_{2}+8 \beta^{2} \delta^{2}\right)^{\frac{1}{2}}\right) \\
& +2\left(\left(2 x_{3}+8 y_{3}\right)^{\frac{1}{2}}+\left(2 x_{3}+8 y_{3}+8 \delta^{2} \lambda^{2}\right)^{\frac{1}{2}}\right) \\
& +2\left(\left(2 x_{4}+8 y_{4}\right)^{\frac{1}{2}}+\left(2 x_{4}+8 y_{4}+8 \delta^{2} \gamma^{2}\right)^{\frac{1}{2}}\right) .
\end{aligned}
$$

where $\rho_{a b c}=\operatorname{Tr}_{d}|\varphi\rangle\left\langle\left.\varphi\right|_{a b c d}, \rho_{a b d}=\operatorname{Tr}_{c} \mid \varphi\right\rangle\left\langle\left.\varphi\right|_{a b c d}, \rho_{a c d}=\right.$ $\operatorname{Tr}_{b}|\varphi\rangle\left\langle\left.\varphi\right|_{a b c d}, \rho_{b c d}=\operatorname{Tr}_{a} \mid \varphi\right\rangle\left\langle\left.\varphi\right|_{a b c d}\right.$, and

$$
\begin{aligned}
& x_{1}=\left(\alpha^{2}+\beta^{2}+\gamma^{2}-\delta^{2}-\lambda^{2}\right)^{2}, \\
& y_{1}=\beta^{2} \gamma^{2}+\alpha^{2} \lambda^{2}+\frac{3}{2} \alpha^{2} \beta^{2}+\gamma^{2} \lambda^{2}+\frac{3}{2} \alpha^{2} \gamma^{2}+\beta^{2} \lambda^{2}, \\
& x_{2}=\left(\alpha^{2}+\beta^{2}-\gamma^{2}+\delta^{2}-\lambda^{2}\right)^{2}, \\
& y_{2}=\beta^{2} \gamma^{2}+\alpha^{2} \lambda^{2}+\frac{3}{2} \alpha^{2} \beta^{2}+\delta^{2} \lambda^{2}+\frac{3}{2} \alpha^{2} \delta^{2}+\delta^{2} \beta^{2}, \\
& x_{3}=\left(\alpha^{2}-\beta^{2}+\gamma^{2}+\delta^{2}-\lambda^{2}\right)^{2}, \\
& y_{3}=\frac{3}{2} \alpha^{2} \delta^{2}+\alpha^{2} \lambda^{2}+\frac{3}{2} \alpha^{2} \gamma^{2}+\delta^{2} \lambda^{2}+\delta^{2} \gamma^{2}+\lambda^{2} \gamma^{2}, \\
& x_{4}=\left(-\alpha^{2}+\beta^{2}+\gamma^{2}+\delta^{2}-\lambda^{2}\right)^{2}, \\
& y_{4}=\frac{3}{2} \beta^{2} \gamma^{2}+\beta^{2} \lambda^{2}+\delta^{2} \gamma^{2}+\delta^{2} \lambda^{2}+\gamma^{2} \lambda^{2}+\frac{3}{2} \delta^{2} \beta \gamma .
\end{aligned}
$$

See proof in Appendix B.

The $n$-qubit Dicke state is an $n$-partite symmetric state defined as $|\mathfrak{D}(n, m)\rangle=\left(\begin{array}{l}n \\ m\end{array}\right)^{-1 / 2} \sum_{P \in \mathcal{P}} P\left(|0\rangle^{\otimes m} \otimes\right.$ $|1\rangle^{\otimes(n-m)}$ ), where $\mathcal{P}$ is the permutation group of $n$ elements. The state $|\mathfrak{D}(4,1)\rangle$ is the standard 4-qubit W state. When $\lambda=0$, the state (24) reduces to the 4 -qubit W-class state:

$$
|\varphi\rangle_{W_{a b c d}}=\alpha|1000\rangle+\beta|0100\rangle+\gamma|0010\rangle+\delta|0001\rangle .
$$

For the state 25$]$ reduces to

$$
\begin{aligned}
& \left\langle S\left(W_{a b c}\right)\right\rangle^{2}+\left\langle S\left(W_{a b d}\right)\right\rangle^{2}+\left\langle S\left(W_{a c d}\right)\right\rangle^{2}+\left\langle S\left(W_{b c d}\right)\right\rangle^{2} \\
& \leq 64\left(1+\alpha^{2} \gamma^{2}+\beta^{2} \delta^{2}+2 \alpha^{2} \beta^{2}+2 \beta^{2} \gamma^{2}+2 \gamma^{2} \delta^{2}\right)
\end{aligned}
$$

where $W_{a b c}, W_{a b d}, W_{a c d}$ and $W_{b c d}$ denote the corresponding reduced states of $|\varphi\rangle\left\langle\left.\varphi\right|_{W_{a b c d}}\right.$.

However, from (8) the violation of Svetlichny operators for tripartite states $W_{a b c}, W_{a b d}, W_{a c d}$ and $W_{b c d}$ satisfy the following relations,

$$
\begin{aligned}
& \left\langle S\left(W_{a b c}\right)\right\rangle \leq 4 \max \left\{\sqrt{4\left(\alpha \beta^{2}+\alpha \gamma^{2}\right)}, \sqrt{8 \beta \gamma^{2}+\left(2 \delta^{2}-1\right)^{2}}\right\}, \\
& \left\langle S\left(W_{a b d}\right)\right\rangle \leq 4 \max \left\{\sqrt{4\left(\alpha \beta^{2}+\alpha \delta^{2}\right)}, \sqrt{8 \beta \delta^{2}+\left(2 \gamma^{2}-1\right)^{2}}\right\}, \\
& \left\langle S\left(W_{a c d}\right)\right\rangle \leq 4 \max \left\{\sqrt{4\left(\alpha \gamma^{2}+\alpha \delta^{2}\right)}, \sqrt{8 \gamma \delta^{2}+\left(2 \beta^{2}-1\right)^{2}}\right\}, \\
& \left\langle S\left(W_{b c d}\right)\right\rangle \leq 4 \max \left\{\sqrt{4\left(\beta \gamma^{2}+\beta \delta^{2}\right)}, \sqrt{8 \gamma \delta^{2}+\left(2 \alpha^{2}-1\right)^{2}}\right\} .
\end{aligned}
$$

Accounting to the fact that $\max \{X, Y\}=\frac{|X-Y|+|X+Y|}{2}$, one has

$$
\begin{aligned}
& \left\langle S\left(W_{a b c}\right)\right\rangle^{2}+\left\langle S\left(W_{a b d}\right)\right\rangle^{2}+\left\langle S\left(W_{a c d}\right)\right\rangle^{2}+\left\langle S\left(W_{b c d}\right)\right\rangle^{2} \\
\leq & 8\left(\left|4\left(\alpha \beta^{2}+\alpha \gamma^{2}\right)-8 \beta \gamma^{2}-\left(2 \delta^{2}-1\right)^{2}\right|\right. \\
& +\left|4\left(\alpha \beta^{2}+\alpha \delta^{2}\right)-8 \beta \delta^{2}-\left(2 \gamma^{2}-1\right)^{2}\right| \\
& +\left|4\left(\beta \gamma^{2}+\beta \delta^{2}\right)-8 \gamma \delta^{2}-\left(2 \alpha^{2}-1\right)^{2}\right| \\
& +\left|4\left(\alpha \gamma^{2}+\alpha \delta^{2}\right)-8 \gamma \delta^{2}-\left(2 \beta^{2}-1\right)^{2}\right| \\
& +8\left(\alpha \beta^{2}+\alpha \gamma^{2}+\alpha \delta^{2}+\frac{3}{2} \beta \gamma^{2}+\frac{3}{2} \beta \delta^{2}+2 \gamma \delta^{2}\right) \\
& \left.+\left(2 \alpha^{2}-1\right)^{2}+\left(2 \beta^{2}-1\right)^{2}+\left(2 \gamma^{2}-1\right)^{2}+\left(2 \delta^{2}-1\right)^{2}\right) .
\end{aligned}
$$

Denote $F$ and $G$ the right sides of $(27)$ and $(29)$, respectively. Figure 3 shows that the value of $F$ is always less than $G$ in the range $\gamma \in[0,1]$ for $\alpha=\beta=0$ and $\delta^{2}=1-\gamma^{2}$.

Equation (27) also gives a kind of trade-off relation among the quantum nonlocality of the reduced states. The maximum value $704 / 7$ of $F$ is attained at $\{\alpha, \beta, \gamma, \delta\}=\{0, \sqrt{2 / 7} \sqrt{3 / 7}, \sqrt{2 / 7}\}$. Figure 4 shows the detailed trade off relations among $\left\langle S_{W_{a b c}}\right\rangle^{2},\left\langle S_{W_{a b d}}\right\rangle^{2}$, $\left\langle S_{W_{a c d}}\right\rangle^{2}$ and $\left\langle S_{W_{b c d}}\right\rangle^{2}$. Here for $\alpha=\beta=0$ and $\delta^{2}=1-\gamma^{2}$, we have $\left\langle S_{W_{a b c}}\right\rangle^{2}=\left\langle S_{W_{a b d}}\right\rangle^{2}$ and $\left\langle S_{W_{a c d}}\right\rangle^{2}=$ $\left\langle S_{W_{b c d}}\right\rangle^{2}$.

\section{CONCLUSIONS}

We have studied the trade-off relationship of genuine tripartite non-locality in a multipartite system. And the corresponding tight upper bounds for GHZ-class states and W-class states have presented, showing that the genuine three-qubit nonlocalities are not independent in a four-qubit system. Meanwhile, we have identified that the reduced three-qubit states of a four-qubit GHZ state can not violate the Svetchlity inequality. Our approach may be also used to investigate the trade off relations of genuine nonlocalities satisfied by the reduced tripartite states of a more general multipartite system.

\section{Acknowledgements}

The work was initiated when the first author was a Phd student in Capital Normal University. LJZ, LC, KW, 


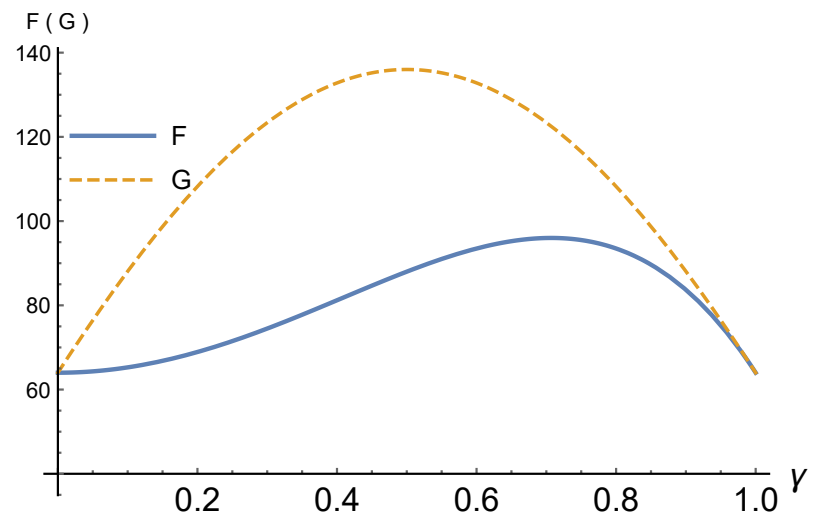

Figure 3: (Color online) In the range of $\gamma \in[0,1]$, the bound $F$ is smaller than $G$.

and YS are supported by the NNSF of China (Grant No. 11871089) and the Fundamental Research Funds for the Central Universities (Grant Nos. KG12080401 and ZG216S1902). SMF and YMG acknowledges the support from NSF of China under Grant No. 11675113 and Key Project of Beijing Municipal Commission of Education (KZ201810028042).
[1] A. Einstein, B. Podolsky, and N. Rosen. Can quantummechanical description of physical reality be considered complete? Phys. Rev., 47:777-780, May 1935.

[2] JOHN S. BELL. On the problem of hidden variables in quantum mechanics. Rev. Mod. Phys., 38:447-452, Jul 1966.

[3] Harry Buhrman, Richard Cleve, Serge Massar, and Ronald de Wolf. Nonlocality and communication complexity. Rev. Mod. Phys., 82:665-698, Mar 2010.

[4] Ashok Ajoy and Pranaw Rungta. Svetlichny's inequality and genuine tripartite nonlocality in three-qubit pure states. Phys. Rev. A, 81:052334, May 2010.

[5] Asher Peres. Classical interventions in quantum systems. ii. relativistic invariance. Phys. Rev. A, 61:022117, Jan 2000.

[6] Li-Jun Zhao, Yu-Min Guo, XianQing Li-Jost, and ShaoMing Fei. Quantum nonlocality can be distributed via separable states. Science China Physics, Mechanics $\&$ Astronomy, 61(7):70321, Mar 2018.

[7] Fernando G. S. L. Brandao, Elizabeth Crosson, M. Burak Sahinoglu Crosson, and John Bowen. Quantum error correcting codes in eigenstates of translation-invariant spin chains. Phys. Rev. Lett., 123:110502, Sep 2019.

[8] Lin Chen, Eric Chitambar, Kavan Modi, and Giovanni Vacanti. Detecting multipartite classical states and their resemblances. Phys. Rev. A, 83:020101, Feb 2011.

[9] Lin Chen and Li Yu. Nonlocal and controlled unitary operators of Schmidt rank three. Phys. Rev. A, 89:062326, Jun 2014.

[10] Lin Chen and Li Yu. Entangling and assisted entangling power of bipartite unitary operations. Phys. Rev. A, 94:022307, Aug 2016.

[11] Lin Chen and Li Yu. Entanglement cost and entangling power of bipartite unitary and permutation operators. Phys. Rev. A, 93:042331, Apr 2016.

[12] Lin Chen and Li Yu. Decomposition of bipartite and multipartite unitary gates into the product of controlled unitary gates. Phys. Rev. A, 91:032308, Mar 2015.

[13] Kai Wang, Lin Chen, Yi Shen, Yize Sun, and Li-Jun Zhao. Constructing $2 \times 2 \times 4$ and $4 \times 4$ unextendible product bases and positive-partial-transpose entangled states. Linear and Multilinear Algebra, 0(0):1-16, 2019.

[14] Lin Chen and Dragomir Z. Djokovic. Qubit-qudit states with positive partial transpose. Phys. Rev. A, 86:062332, Dec 2012.

[15] Thiago R. de Oliveira, A. Saguia, and M. S. Sarandy. Nonviolation of bells inequality in translation invariant systems. EPL (Europhysics Letters), 100(6):60004, dec 2012.

[16] Zhao Yu Sun, Yu Ying Wu, Hai Lin Huang, Bo Jun Chen, and Bo Wang. Violation of bell inequality in perfect translation invariant systems. Physical Review A, 88(88):340-347, 2013.

[17] Marcin Pawlowski and Časlav Brukner. Monogamy of bell's inequality violations in nonsignaling theories. Phys. Rev. Lett., 102:030403, Jan 2009.

[18] Ming Li, Zong Wang, Jing Wang, Shuqian Shen, and Shao ming Fei. The norms of bloch vectors and classification of four-qudits quantum states. EPL (Europhysics Letters ), 125(2):20006, feb 2019

[19] Ming-Xing Luo. Nonlocality of all quantum networks. Phys. Rev. A, 98:042317, Oct 2018.

[20] R. Augusiak, M. Demianowicz, and J. Tura. Constructing genuinely entangled multipartite states with applications to local hidden variables and local hidden states models. Phys. Rev. A, 98:012321, Jul 2018.

[21] Christopher Eltschka and Jens Siewert. Entanglement of three-qubit greenberger-horne-zeilinger-symmetric states. Phys. Rev. Lett., 108:020502, Jan 2012.

[22] Biswajit Paul, Kaushiki Mukherjee, and Debasis Sarkar. Nonlocality of three-qubit greenberger-horne-zeilingersymmetric states. Phys. Rev. A, 94:032101, Sep 2016.

[23] S. Ghose, S. Debnath, N. Sinclair, A. Kabra, and R. Stock. Multiqubit nonlocality in families of 3- and 


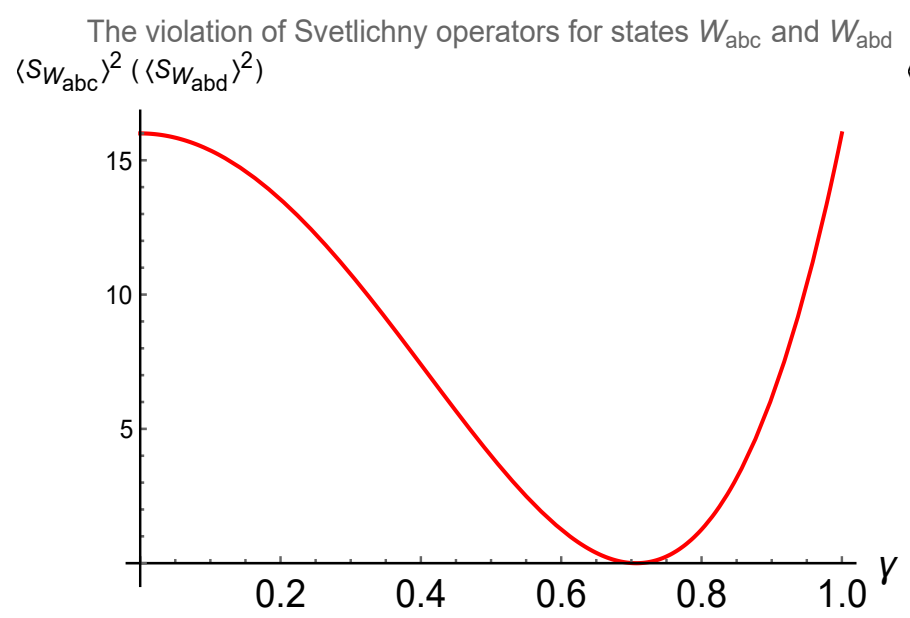

The violation of Svetlichny operators for states $W_{\text {acd }}$ and $W_{\text {bcd }}$ $\left\langle S_{W_{\text {acd }}}\right\rangle^{2}\left(\left\langle S_{W_{\mathrm{bcd}}}\right\rangle^{2}\right)$

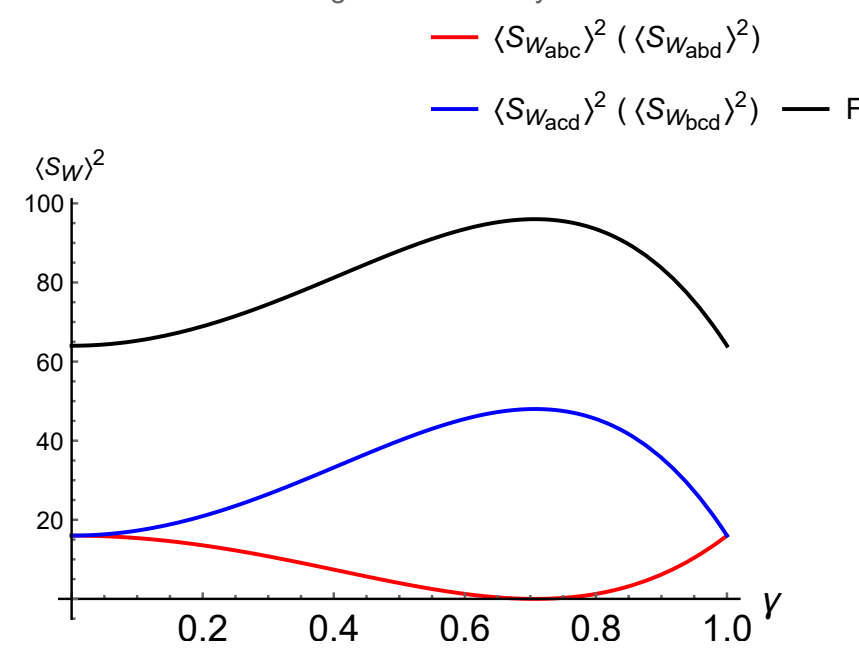

Figure 4: (Color online) In $\gamma \in[0,1]$, the quantities $\left\langle S_{W_{a b c}}\right\rangle^{2}\left(=\left\langle S_{W_{a b d}}\right\rangle^{2}\right)$ and $\left\langle S_{W_{a c d}}\right\rangle^{2}\left(=\left\langle S_{W_{b c d}}\right\rangle^{2}\right)$ vary in a way such that their summation kept to be bounded by $F$.

4-qubit entangled states. Journal of Physics A Mathematical \&5 Theoretical, 43(44):445301, 2010.

[24] Swapan Rana and Preeti Parashar. Optimal reducibility of all $w$ states equivalent under stochastic local operations and classical communication. Phys. Rev. A, 84:052331, Nov 2011.

[25] Ravishankar Ramanathan and Piotr Mironowicz. Tradeoffs in multiparty bell-inequality violations in qubit networks. Phys. Rev. A, 98:022133, Aug 2018.

[26] P. Kurzynski, T. Paterek, R. Ramanathan, W. Laskowski, and D. Kaszlikowski. Monogamy of multipartite bell inequality violations. Physical Review Letters, 106(106):180402, 2010.

[27] John F. Clauser, Michael A. Horne, Abner Shimony, and Richard A. Holt. Proposed experiment to test local hidden-variable theories. Phys. Rev. Lett., 23:880-884, 1969.

[28] R. Horodecki, P. Horodecki, and M. Horodecki. Violating Bell inequality by mixed spin- $1 / 2$ states: necessary and sufficient condition. Phys. Lett. A, 200(5):340-344, 1995.

[29] R. Horodecki. Two-spin-1/2 mixtures and Bell's inequal- ities. Phys. Lett. A, 210(4):223-226, 1996.

[30] HuiHui Qin, ShaoMing Fei, and Xianqing Li-Jost. Tradeoff relations of Bell violations among pairwise qubit systems. Phys. Rev. A, 92:062339, 2015.

[31] Shuming Cheng and Michael J. W. Hall. Anisotropic invariance and the distribution of quantum correlations. Phys. Rev. Lett., 118:010401, 2017.

[32] Jean-Daniel Bancal, Jonathan Barrett, Nicolas Gisin, and Stefano Pironio. Definitions of multipartite nonlocality. Phys. Rev. A, 88:014102, Jul 2013.

[33] Liang Qiu. Nonlocality sudden birth and transfer in system and environment. Chinese Physics Letters, 28(3):030301, mar 2011.

[34] Ming Li, Shuqian Shen, Naihuan Jing, ShaoMing Fei, and Xianqing Li-Jost. Tight upper bound for the maximal quantum value of the Svetlichny operators. Phys. Rev. A, 96:042323, 2017.

[35] George Svetlichny. Distinguishing three-body from twobody nonseparability by a Bell-type inequality. Phys. Rev. D, 35:3066-3069, 1987.

[36] Peter Mitchell, Sandu Popescu, and David Roberts. Con- 
ditions for the confirmation of three-particle nonlocality. Phys. Rev. A, 70:060101, 2004.

[37] S. Ghose, N. Sinclair, S. Debnath, P. Rungta, and R. Stock. Tripartite entanglement versus tripartite nonlocality in three-qubit Greenberger-Horne-Zeilinger-Class states. Phys. Rev. Lett., 102:250404, 2009.

\section{Appendix A: Proof of Theorem 2}

For the reduced state $\Phi_{a b c}$, one has the expectation value of the Svetlichny operator,

$$
\langle A D C\rangle_{\Phi_{a b c}}=\cos \theta \sin \theta_{a} \sin \theta_{c} \sin \theta_{d} \cos \left(\phi_{a}+\phi_{c}+\phi_{d}\right)
$$

$\left\langle A D^{\prime} C^{\prime}\right\rangle_{\Phi_{a b c}},\left\langle A^{\prime} D^{\prime} C\right\rangle_{\Phi_{a b c}}$ and $\left\langle A^{\prime} D C^{\prime}\right\rangle_{\Phi_{a b c}}$ have similar expressions. Therefore we have

$$
\begin{aligned}
\left\langle S\left(\Phi_{a b c}\right)\right\rangle= & 2\left|\cos \omega\langle A D C\rangle_{\Phi_{a b c}}+\sin \omega\left\langle A D^{\prime} C^{\prime}\right\rangle_{\Phi_{a b c}}+\sin \omega\left\langle A^{\prime} D^{\prime} C\right\rangle_{\Phi_{a b c}}-\cos \omega\left\langle A^{\prime} D C^{\prime}\right\rangle_{\Phi_{a b c}}\right| \\
\leq & 2\left|\left(\langle A D C\rangle_{\Phi_{a b c}}^{2}+\left\langle A D^{\prime} C^{\prime}\right\rangle_{\Phi_{a b c}}^{2}\right)^{1 / 2}+\left(\left\langle A^{\prime} D^{\prime} C\right\rangle_{\Phi_{a b c}}^{2}+\left\langle A^{\prime} D C^{\prime}\right\rangle_{\Phi_{a b c}}^{2}\right)^{1 / 2}\right| \\
\leq & 2 \mid\left\{\left(\cos \theta \sin \theta_{a} \sin \theta_{c} \sin \theta_{d} \cos \left(\phi_{a}+\phi_{c}+\phi_{d}\right)\right)^{2}+\left(\cos \theta \sin \theta_{a} \sin \theta_{c^{\prime}} \sin \theta_{d^{\prime}} \cos \left(\phi_{c^{\prime}}+\phi_{d^{\prime}}+\phi_{a}\right)\right)^{2}\right\}^{1 / 2} \\
& +\left\{\left(\cos \theta \sin \theta_{c} \sin \theta_{a^{\prime}} \sin \theta_{d^{\prime}} \cos \left(\phi_{a^{\prime}}+\phi_{d^{\prime}}+\phi_{c}\right)\right)^{2}+\left(\cos \theta \cos \omega \sin \theta_{d} \sin \theta_{a^{\prime}} \sin \theta_{c^{\prime}} \cos \left(\phi_{a^{\prime}}+\phi_{c^{\prime}}+\phi_{d}\right)\right)^{2}\right\}^{1 / 2} \mid \\
\leq & 2\left|\left(\cos ^{2} \theta \sin ^{2} \theta_{d}+\cos ^{2} \theta \sin ^{2} \theta_{d^{\prime}}\right)^{1 / 2}+\left(\cos ^{2} \theta \sin ^{2} \theta_{d^{\prime}}+\cos ^{2} \theta \sin ^{2} \theta_{d}\right)^{1 / 2}\right| \\
\leq & 4\left|\cos \theta\left(\sin ^{2} \theta_{d}+\sin ^{2} \theta_{d^{\prime}}\right)^{1 / 2}\right| \\
\leq & 4 \sqrt{2}|\cos \theta|
\end{aligned}
$$

When $\phi_{i}+\phi_{j}+\phi_{k}=0$, where $i \in\left\{a, a^{\prime}\right\}, j \in\left\{d, d^{\prime}\right\}$ and $k \in\left\{c, c^{\prime}\right\}$, one has $\left\langle S\left(\Phi_{a b c}\right)\right\rangle=4 \sqrt{2}|\cos \theta|$.

For the reduced state $\Phi_{a b d}$, we have

$$
\langle A D C\rangle_{\Phi_{a b d}}=\frac{1}{2} \cos \theta_{a} \cos \theta_{d}\left(\sin 2 \theta \sin \theta_{c} \cos \phi_{c}+2 \cos ^{2} \theta \cos \theta_{c}\right)
$$

The expressions for $\left\langle A D^{\prime} C^{\prime}\right\rangle_{\Phi_{a b d}},\left\langle A^{\prime} D^{\prime} C\right\rangle_{\Phi_{a b d}}$ and $\left\langle A^{\prime} D C^{\prime}\right\rangle_{\Phi_{a b d}}$ are similar. By direct computation we obtain

$$
\begin{aligned}
\left\langle S\left(\Phi_{a b d}\right)\right\rangle= & 2\left|\cos \omega\langle A D C\rangle_{\Phi_{a b d}}+\sin \omega\left\langle A D^{\prime} C^{\prime}\right\rangle_{\Phi_{a b d}}+\sin \omega\left\langle A^{\prime} D^{\prime} C\right\rangle_{\Phi_{a b d}}-\cos \omega\left\langle A^{\prime} D C^{\prime}\right\rangle_{\Phi_{a b d}}\right| \\
\leq & 2\left|\left(\langle A D C\rangle_{\Phi_{a b d}}^{2}+\left\langle A D^{\prime} C^{\prime}\right\rangle_{\Phi_{a b d}}^{2}\right)^{1 / 2}+\left(\left\langle A^{\prime} D^{\prime} C\right\rangle_{\Phi_{a b d}}^{2}+\left\langle A^{\prime} D C^{\prime}\right\rangle_{\Phi_{a b d}}^{2}\right)^{1 / 2}\right| \\
= & 2 \mid\left\{\left(\frac{1}{2} \cos \theta_{a} \cos \theta_{d}\left(\sin 2 \theta \sin \theta_{c} \cos \phi_{c}+2 \cos ^{2} \theta \cos \theta_{c}\right)\right)^{2}\right. \\
& \left.+\left(\frac{1}{2} \cos \theta_{a} \cos \theta_{d^{\prime}}\left(\sin 2 \theta \sin \theta_{c^{\prime}} \cos \phi_{c^{\prime}}+2 \cos ^{2} \theta \cos \theta_{c^{\prime}}\right)\right)^{2}\right\}^{1 / 2} \\
& +\left\{\left(\frac{1}{2} \cos \theta_{a^{\prime}} \cos \theta_{d^{\prime}}\left(\sin 2 \theta \sin \theta_{c} \cos \phi_{c}+2 \cos ^{2} \theta \cos \theta_{c}\right)\right)^{2}\right. \\
& \left.+\left(\frac{1}{2} \cos \theta_{d} \cos \theta_{a^{\prime}}\left(\sin 2 \theta \sin \theta_{c^{\prime}} \cos \phi_{c^{\prime}}+2 \cos ^{2} \theta \cos \theta_{c^{\prime}}\right)\right)^{2}\right\}^{1 / 2} \mid \\
\leq & 4\left|\left(\frac{1}{4} \sin ^{2} 2 \theta+\sin 2 \theta \cos ^{2} \theta+\cos ^{4} \theta\right)^{1 / 2}\right| \\
\leq & 4\left|\cos ^{2} \theta+\frac{1}{2} \sin 2 \theta\right| .
\end{aligned}
$$

Taking into account that $\left\langle S\left(\Phi_{a b d}\right)\right\rangle=\left\langle S\left(\Phi_{a c d}\right)\right\rangle=\left\langle S\left(\Phi_{b c d}\right)\right\rangle$, one proves the Theorem. 


\section{Appendix B: Proof of Theorem 3}

For the reduced state $\rho_{a b c}$,

$$
\begin{aligned}
\rho_{a b c}= & \operatorname{Tr}_{d}|\varphi\rangle\left\langle\left.\varphi\right|_{a b c d}\right. \\
= & \alpha^{2}|100\rangle\langle 100|+\alpha \beta| 100\rangle\langle 010|+\alpha \gamma| 100\rangle\langle 001| \\
& +\alpha \lambda|100\rangle\langle 000|+\alpha \beta| 010\rangle\left\langle 100\left|+\beta^{2}\right| 010\right\rangle\langle 010| \\
& +\beta \gamma|010\rangle\langle 001|+\beta \lambda| 010\rangle\langle 000|+\alpha \gamma| 001\rangle\langle 100| \\
& +\beta \gamma|001\rangle\left\langle 010\left|+\gamma^{2}\right| 001\right\rangle\langle 001|+\gamma \lambda| 001\rangle\langle 000| \\
& +\delta^{2}|000\rangle\langle 000|+\alpha \lambda| 000\rangle|100\rangle+\beta \lambda|000\rangle\langle 010| \\
& +\gamma \lambda|000\rangle\left\langle 001\left|+\lambda^{2}\right| 000\right\rangle\langle 000| .
\end{aligned}
$$

we can obtain

$$
\begin{aligned}
& \langle A D C\rangle_{\rho_{a b c}} \\
= & -\left(\alpha^{2}+\beta^{2}+\gamma^{2}-\sigma^{2}-\lambda^{2}\right) \cos \theta_{a} \cos \theta_{c} \cos \theta_{d} \\
& +2 \beta \gamma \cos \left(\phi_{c}-\phi_{d}\right) \cos \theta_{a} \sin \theta_{c} \sin \theta_{d} \\
& +2 \alpha \lambda \cos \phi_{a} \sin \theta_{a} \cos \theta_{c} \cos \theta_{d} \\
& +2 \alpha \beta \cos \left(\phi_{a}-\phi_{d}\right) \sin \theta_{a} \cos \theta_{c} \sin \theta_{d} \\
& +2 \gamma \lambda \cos \phi_{c} \cos \theta_{a} \sin \theta_{c} \cos \theta_{d} \\
& +2 \alpha \gamma \cos \left(\phi_{a}-\phi_{c}\right) \sin \theta_{a} \sin \theta_{c} \cos \theta_{d} \\
& +2 \beta \lambda \cos \phi_{d} \cos \theta_{a} \cos \theta_{c} \sin \theta_{d} .
\end{aligned}
$$

Let

$$
\begin{aligned}
& u_{1}=-\left(\alpha^{2}+\beta^{2}+\gamma^{2}-\sigma^{2}-\lambda^{2}\right), \quad v_{1}=0, \\
& u_{2}=2 \beta \gamma \cos \left(\phi_{c}-\phi_{d}\right), \quad v_{2}=2 \alpha \lambda \cos \phi_{a}, \\
& u_{3}=2 \alpha \beta \cos \left(\phi_{a}-\phi_{d}\right), \quad v_{3}=2 \gamma \lambda \cos \phi_{c}, \\
& u_{4}=2 \alpha \gamma \cos \left(\phi_{a}-\phi_{c}\right), \quad v_{4}=2 \beta \lambda \cos \phi_{d},
\end{aligned}
$$

and

$$
\begin{aligned}
& x_{1}=\cos \theta_{a} \cos \theta_{c} \cos \theta_{d}, \quad y_{1}=\sin \theta_{a} \sin \theta_{c} \sin \theta_{d}, \\
& x_{2}=\cos \theta_{a} \sin \theta_{c} \sin \theta_{d}, \quad y_{2}=\sin \theta_{a} \cos \theta_{c} \cos \theta_{d}, \\
& x_{3}=\sin \theta_{a} \cos \theta_{c} \sin \theta_{d}, \quad y_{3}=\cos \theta_{a} \sin \theta_{c} \cos \theta_{d}, \\
& x_{4}=\sin \theta_{a} \sin \theta_{c} \cos \theta_{d}, \quad y_{4}=\cos \theta_{a} \cos \theta_{c} \sin \theta_{d} .
\end{aligned}
$$

One can verify that $\sum_{j=1}^{4}\left(x_{j}^{2}+y_{j}^{2}\right)=1$. Hence we consider the following optimization:

$$
\max \left(\sum_{i} u_{i} x_{i}+\sum_{j} v_{j} y_{j}\right) \quad \text { s.t. } \quad \sum_{j=1}^{4}\left(x_{j}^{2}+y_{j}^{2}\right)=1 .
$$

Using Lagrange multiplier, we have the maximum $k=\sqrt{\sum_{i}\left(u_{i}^{2}+v_{i}^{2}\right)}$. It follows that the maximum is attained when each $\cos \phi= \pm 1$.

Therefore, we have

$$
\langle A D C\rangle_{\rho_{a b c}} \leq \sqrt{\left(\alpha^{2}+\beta^{2}+\gamma^{2}-\sigma^{2}-\lambda^{2}\right)^{2}+4\left(\beta^{2} \gamma^{2}+\alpha^{2} \lambda^{2}+\alpha^{2} \beta^{2}+\gamma^{2} \lambda^{2}+\alpha^{2} \gamma^{2}+\beta^{2} \lambda^{2}\right)} .
$$


Similarly, we have

$$
\begin{aligned}
& \left\langle A D^{\prime} C^{\prime}\right\rangle_{\rho_{a b c}} \leq \sqrt{\left(\alpha^{2}+\beta^{2}+\gamma^{2}-\delta^{2}-\lambda^{2}\right)^{2}+4\left(\beta^{2} \gamma^{2}+\alpha^{2} \lambda^{2}+2 \alpha^{2} \beta^{2}+\gamma^{2} \lambda^{2}+2 \alpha^{2} \gamma^{2}+\beta^{2} \lambda^{2}\right)}, \\
& \left\langle A^{\prime} D^{\prime} C\right\rangle_{\rho_{a b c}} \leq \sqrt{\left(\alpha^{2}+\beta^{2}+\gamma^{2}-\delta^{2}-\lambda^{2}\right)^{2}+4\left(2 \beta^{2} \gamma^{2}+\alpha^{2} \lambda^{2}+\alpha^{2} \beta^{2}+\gamma^{2} \lambda^{2}+2 \alpha^{2} \gamma^{2}+\beta^{2} \lambda^{2}\right)}, \\
& \left\langle A^{\prime} D C^{\prime}\right\rangle_{\rho_{a b c}} \leq \sqrt{\left(\alpha^{2}+\beta^{2}+\gamma^{2}-\delta^{2}-\lambda^{2}\right)^{2}+4\left(2 \beta^{2} \gamma^{2}+\alpha^{2} \lambda^{2}+2 \alpha^{2} \beta^{2}+\gamma^{2} \lambda^{2}+\alpha^{2} \gamma^{2}+\beta^{2} \lambda^{2}\right)} .
\end{aligned}
$$

Therefore, concerning the violation of the Svetlichny inequality with respect to the reduced state $\rho_{a b c}$ we have

$$
\begin{aligned}
\left\langle S\left(\rho_{a b c}\right)\right\rangle= & 2 \mid \cos \theta\langle A D C\rangle_{\rho_{a b c}}+\sin \theta\left\langle A D^{\prime} C^{\prime}\right\rangle_{\rho_{a b c}} \\
& +\sin \theta\left\langle A^{\prime} D^{\prime} C\right\rangle_{\rho_{a b c}}-\cos \theta\left\langle A^{\prime} D C^{\prime}\right\rangle_{\rho_{a b c}} \mid \\
\leq & 2 \mid\left(\langle A D C\rangle_{\rho_{a b c}}^{2}+\left\langle A D^{\prime} C^{\prime}\right\rangle_{\rho_{a b c}}^{2}\right)^{1 / 2} \\
& +\left(\left\langle A^{\prime} D^{\prime} C\right\rangle_{\rho_{a b c}}^{2}+\left\langle A^{\prime} D C^{\prime}\right\rangle_{\rho_{a b c}}^{2}\right)^{1 / 2} \mid \\
= & 2\left(\left(2 x_{1}+8 y_{1}\right)^{\frac{1}{2}}+\left(2 x_{1}+8 y_{1}+8 \beta^{2} \gamma^{2}\right)^{\frac{1}{2}}\right),
\end{aligned}
$$

where $x_{1}=\left(\alpha^{2}+\beta^{2}+\gamma^{2}-\delta^{2}-\lambda^{2}\right)^{2}, y_{1}=\beta^{2} \gamma^{2}+\alpha^{2} \lambda^{2}+\frac{3}{2} \alpha^{2} \beta^{2}+\gamma^{2} \lambda^{2}+\frac{3}{2} \alpha^{2} \gamma^{2}+\beta^{2} \lambda^{2}$. Similarly, with respect to the reduced states $\rho_{a b d}, \rho_{a c d}$ and $\rho_{b c d}$, we get

$$
\left\langle S\left(\rho_{a b d}\right)\right\rangle \leq 2\left(\left(2 x_{2}+8 y_{2}\right)^{\frac{1}{2}}+\left(2 x_{2}+8 y_{2}+8 \beta^{2} \delta^{2}\right)^{\frac{1}{2}}\right),
$$

where $x_{2}=\left(\alpha^{2}+\beta^{2}-\gamma^{2}+\delta^{2}-\lambda^{2}\right)^{2}, y_{2}=\beta^{2} \gamma^{2}+\alpha^{2} \lambda^{2}+\frac{3}{2} \alpha^{2} \beta^{2}+\delta^{2} \lambda^{2}+\frac{3}{2} \alpha^{2} \delta^{2}+\delta^{2} \beta^{2}$.

$$
\left\langle S\left(\rho_{a c d}\right)\right\rangle \leq 2\left(\left(2 x_{3}+8 y_{3}\right)^{\frac{1}{2}}+\left(2 x_{3}+8 y_{3}+8 \delta^{2} \lambda^{2}\right)^{\frac{1}{2}}\right),
$$

where $x_{3}=\left(\alpha^{2}-\beta^{2}+\gamma^{2}+\delta^{2}-\lambda^{2}\right)^{2}, y_{3}=\frac{3}{2} \alpha^{2} \delta^{2}+\alpha^{2} \lambda^{2}+\frac{3}{2} \alpha^{2} \gamma^{2}+\delta^{2} \lambda^{2}+\delta^{2} \gamma^{2}+\lambda^{2} \gamma^{2}$.

$$
\left\langle S\left(\rho_{b c d}\right)\right\rangle \leq 2\left(\left(2 x_{4}+8 y_{4}\right)^{\frac{1}{2}}+\left(2 x_{4}+8 y_{4}+8 \delta^{2} \gamma^{2}\right)^{\frac{1}{2}}\right),
$$

where $x_{4}=\left(-\alpha^{2}+\beta^{2}+\gamma^{2}+\delta^{2}-\lambda^{2}\right)^{2}, y_{4}=\frac{3}{2} \beta^{2} \gamma^{2}+\beta^{2} \lambda^{2}+\delta^{2} \gamma^{2}+\delta^{2} \lambda^{2}+\gamma^{2} \lambda^{2}+\frac{3}{2} \delta^{2} \beta \gamma$. 